\title{
Fitting of a New Design of Full Scleral Contact Lens in Advanced Keratoconus with Previous Implantation of Intracorneal Ring Segments
}

\author{
David P Piñero Llorens
}

\begin{abstract}
This case report reports the visual rehabilitation obtained with the fitting of a new design of full scleral contact lens (ICD 16.5 contact lens, Paragon Vision Sciences, distributed by Lenticon, Madrid, Spain) in a cornea with advanced keratoconus and previous implantation of intracorneal ring segment with a very limited effect. This eye had a refraction of $-3.00 \times 55^{\circ}$ cylinder, providing a visual acuity of 0.5 LogMAR. The topographic pattern was very irregular with the presence of a significant central protrusion and a significant central corneal thinning. Some previous unsuccessful fittings have been performed with corneal and corneal-scleral lenses. A comfortable wearing was achieved with a fully scleral contact lens of $4600 \mu \mathrm{m}$ of sagittal height, optical power of $-11.25 \mathrm{D}$, and providing an apical clearance of $196 \mu \mathrm{m}$. A visual acuity of 0.0 LogMAR combined with a relevant aberrometric improvement was achieved with this contact lens. The patient was completely satisfied with the fitting. The result was maintained during 1 year after the fitting. Full scleral lenses are then able to provide comfortable wear and a significant increase in visual acuity combined with a significant improvement in the visual quality in eyes with advanced keratoconus.
\end{abstract}

Keywords: Contact lenses, Corneal irregularity corneal asymmetry, Intrastromal corneal rings, Keratoconus, Sirius.

How to cite this article: Llorens DPP. Fitting of a New Design of Full Scleral Contact Lens in Advanced Keratoconus with Previous Implantation of Intracorneal Ring Segments. Int J Kerat Ect Cor Dis 2015;4(2):56-59.

Source of support: Nil

Conflict of interest: None

\section{INTRODUCTION}

Typically, scleral lenses have been defined as any type of contact lens that rest at least in part on the sclera. ${ }^{1}$ However, two groups should be differentiated depending if there is or not corneal touch: cornea-scleral lenses that

\section{Assistant Professor}

Department of Optics, Pharmacology and Anatomy, University of Alicante, Alicante, Spain

Corresponding Author: David P Piñero Llorens, Assistant Professor, Department of Optics, Pharmacology and Anatomy University of Alicante, Alicante, Spain, Phone: 34965903500 e-mail: david.pinyero@ua.es partly rests on the cornea (centrally or peripherally) and partly on the sclera (diameters between 12.5 and $15 \mathrm{~mm}$ ), and full scleral lenses that rest entirely on the sclera (diameters of more than $15 \mathrm{~mm}$ ). ${ }^{1}$ This last type of lenses have been typically considered as an appropriate option for the correction of irregular astigmatism (postLASIK irregularity, post-keratoplasty, keratoconus) as it is neutralized with the tear meniscus formed between the cornea and the contact lens. ${ }^{2}$ Furthermore, as full scleral lenses only rest on the sclera and have a large diameter, they provide great comfort due to the lower sensitivity of the conjunctiva and the less interaction with the eyelid. ${ }^{2}$ However, in spite of these potential benefits, there are to date a low number of studies evaluating the outcomes of the new designs developed of this type of contact lenses. ${ }^{3-6}$ This case report shows the clinical outcome obtained with a new design of full scleral contact lens (ICD 16.5 contact lens, Paragon Vision Sciences, distributed by Lenticon, Madrid, Spain) in a cornea with advanced keratoconus and previous implantation of intracorneal ring segment with a very limited effect.

\section{CASE REPORT}

A 26-year-old man visited our Contactology Unit of the Ophthalmology Department (Oftalmar) of the Medimar International Hospital in Alicante in October 2013 with a medical report of all his previous ocular surgical procedures and treatments. The patient had been diagnosed with keratoconus in both eyes several years ago. Three years before our visit, both eyes were implanted with intracorneal ring segments (KeraRing, Mediphacos, Belo Horizonte, Brazil). The visual result obtained with left eye (LE) was very satisfactory, achieving an uncorrected visual acuity (UDVA) of 0.0 LogMAR. However, the visual outcome in the right eye (RE) was very poor and the use of special contact lenses was recommended. After performing some tries with different contact lens designs (only tolerated 3 or 4 hours), he attended to our clinic asking for a new design allowing him to increase the wearing time.

On our clinical examination, the RE presented a subjective refraction of $-3.00 \times 55^{\circ}$ cylinder, providing a visual acuity of $0.5 \log$ MAR. The UDVA was 0.6 
LogMAR. On slitlamp examination, the cornea showed Vogt striae as well as a mild central leukoma (Fig. 1). Intracorneal ring segments were clearly visible, with no significantly asymmetric position (Fig. 1). A complete evaluation of the corneal structure was performed with the Scheimpflug photography-based system Sirius (CSO, Firenze, Italy). Figure 2 shows the corneal thickness map, tangential map of the anterior corneal surface, and elevation maps of anterior and posterior corneal surfaces of RE. As shown, a very irregular topographic pattern with the presence of a significant central protrusion was found as well as a significant central corneal thinning. Mean central corneal thickness in this eye was $355 \mu \mathrm{m}$. Mean anterior and posterior keratometric readings were $64.71 / 73.82 / 22^{\circ}$ and $-7.94 /-14.92 / 23^{\circ}$, respectively. An

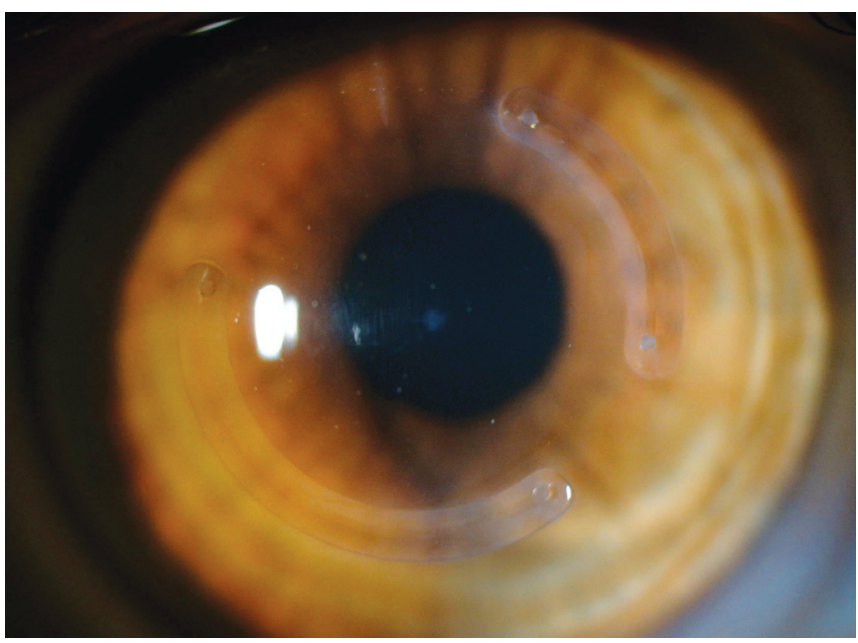

Fig. 1: Frontal image obtained with a slit-lamp biomicroscope of the keratoconus cornea analyzed in the current case report

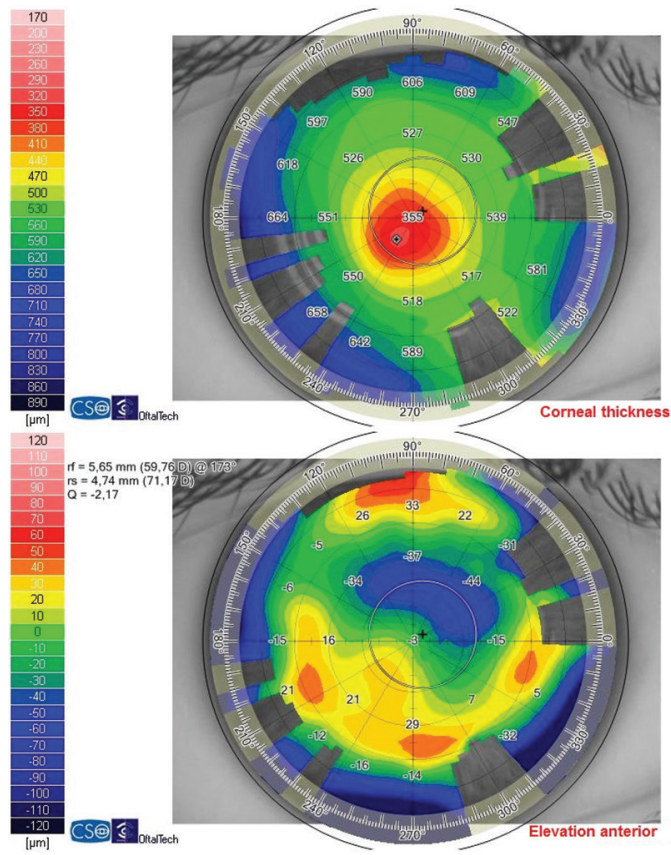

ocular aberrometric analysis was also performed with the iTrace system (Tracey Technologies Corp., Houston, Texas, USA). The following aberrometric data was obtained for a $5 \mathrm{~mm}$ pupil: higher order aberration (HOA) root mean square (RMS) of $7.09 \mu \mathrm{m}$, primary coma $\left(\mathrm{Z}_{3}{ }^{ \pm 1}\right) \mathrm{RMS}$ of $6.58 \mu \mathrm{m}$, primary spherical aberration Zernike term $\left(Z_{4}{ }^{0}\right)$ of $-0.06 \mu \mathrm{m}$ and a trefoil $\left(\mathrm{Z}_{3}{ }^{ \pm 3}\right) \mathrm{RMS}$ of $0.85 \mu \mathrm{m}$.

Due to the high level of irregularity of the cornea evaluated, a full scleral contact lens, and then only resting on the sclera was fitted. Specifically, the ICD 16.5 contact lens (Irregular Corneal Design) was fitted (Paragon Vision Sciences, distributed by Lenticon, Madrid, Spain) that has four differentiated zones allowing a correct centration with no corneal touch (Fig. 3). These zones are: central clearance zone (CCZ), peripheral central clearance zone (PCCZ), limbal clearance zone (LCZ), and scleral landing zone (SLZ) (Fig. 3). This contact lens is fitted considering the sagittal height instead of keratometry. This lens is a rigid permeable to gas (RGP) contact lens manufactured in material HDS 100 from Paragon Vision Sciences (USA). This material is a thermoset fluorosilicone acrylate copolymer derived primarily from siloxane acrylate, trifluoroethyl methacrylate and methylmethacrylate with a water content of less than $1 \%$ (Paflufocon D), with a Dk (oxygen permeability) of 100 Fatt.

According to the manufacturer, a central apical clearance between 300 and $400 \mu \mathrm{m}$ must be present for an adequate fitting. In our case, after several tries, the most comfortable and stable fitting was achieved with a contact lens of $4600 \mu \mathrm{m}$ of sagittal height and optical

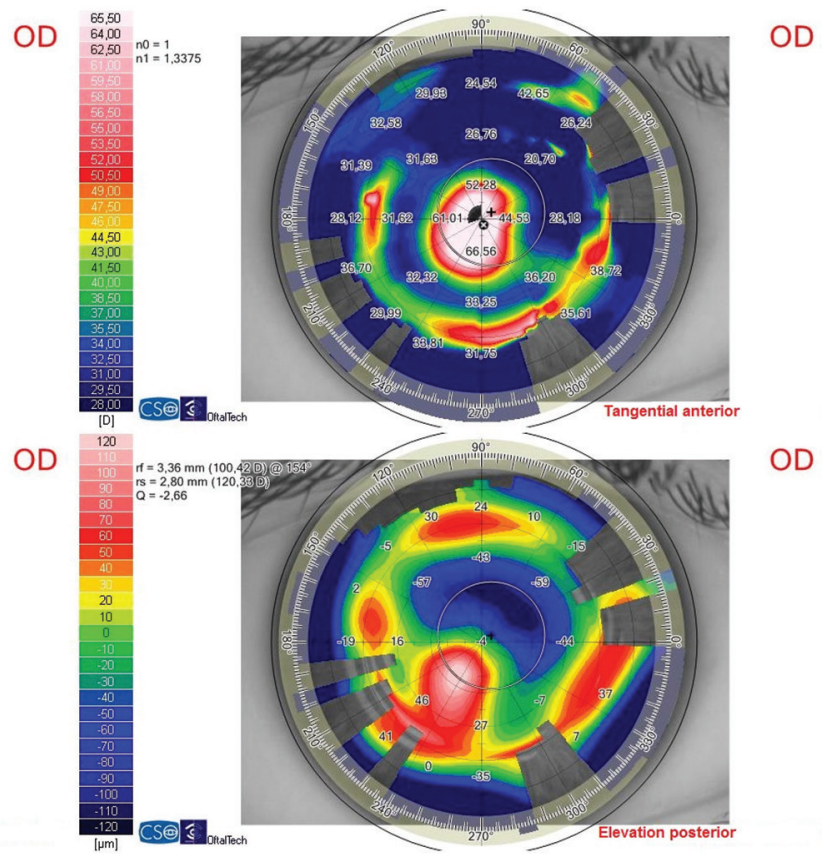

Fig. 2: Evaluation of the corneal structure by means of the Scheimpflug photography-based system Sirius (CSO, Firenze, Italy). Top left: Corneal thickness map; Top right: anterior tangential topographic map; Low left: elevation anterior topographic map; Low right: elevation posterior topographic map 
power of $-11.25 \mathrm{D}$. This lens provided an apical clearance of $196 \mu \mathrm{m}$ as measured by spectral-domain optical coherence tomography (OCT 3D-1000 system, Topcon) (Fig. 4). The contact lens was well-centered with an appropriate scleral resting (Fig. 5). A visual acuity of 0.0 LogMAR was achieved with this contact lens and the patient was completely satisfied with the fitting. Specifically, the patient referred a very significant improvement of the visual quality of this eye which was confirmed with the post-fitting aberrometric analysis obtained for a $5 \mathrm{~mm}$ pupil: HOA RMS of $1.26 \mu \mathrm{m}$, primary coma $\left(\mathrm{Z}_{3}{ }^{ \pm 1}\right) \mathrm{RMS}$ of $0.59 \mu \mathrm{m}$, primary spherical aberration Zernike term $\left(\mathrm{Z}_{4}{ }^{0}\right)$ of $-0.03 \mu \mathrm{m}$ and a trefoil $\left(\mathrm{Z}_{3}{ }^{ \pm 3}\right) \mathrm{RMS}$ of $0.22 \mu \mathrm{m}$. At 1 year after the fitting, the patient maintains a comfortable wearing and the same level of visual quality.

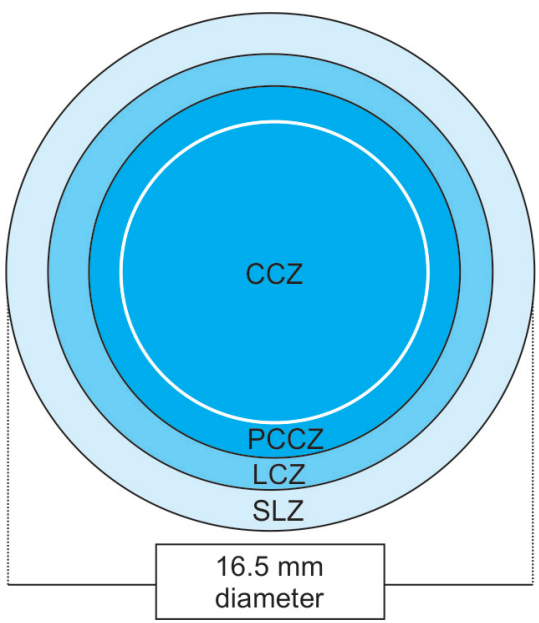

Fig. 3: The design of the contact lens fitted in the current case report

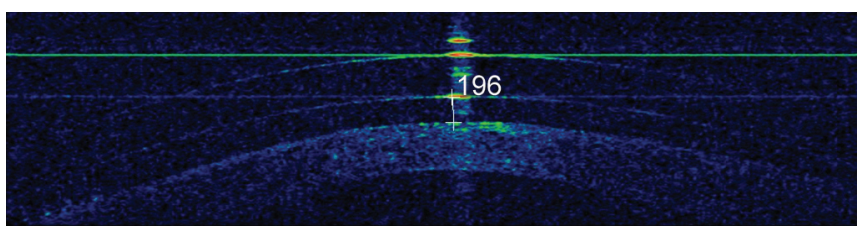

Fig. 4: Visualization of the apical clearance by means of spectraldomain optical coherence tomography of the scleral contact lens fitted

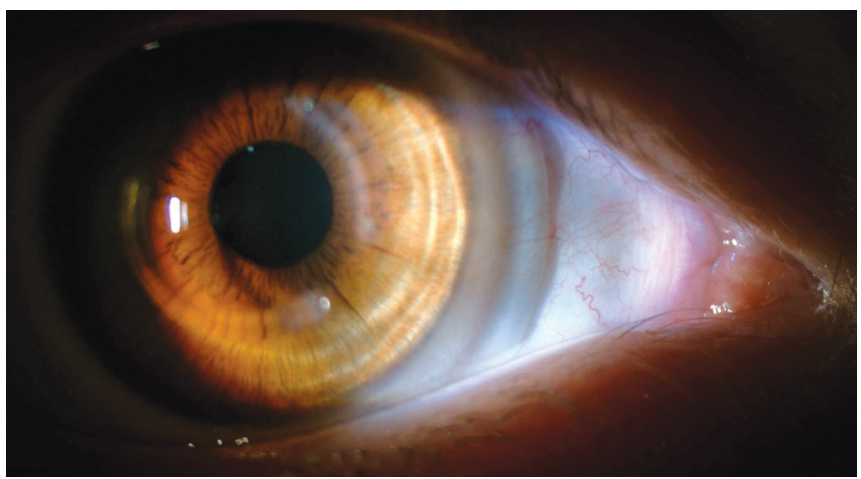

Fig. 5: Frontal image obtained with a slit-lamp biomicroscope of the contact lens fitted in the advanced keratoconus eye evaluated in the current case report

\section{DISCUSSION}

This case report shows the clear applicability of the new designs of full scleral contact lenses in corneas with high levels of irregularity. Specifically, we show the application of this type of contact lens for achieving a complete visual rehabilitation in an advanced keratoconus eye. To this date, some studies have been published showing the potential of full scleral lenses in irregular corneas. Pullum et $\mathrm{al}^{2}$ analyzed a database of 1003 patients (1560 eyes) either assessed for scleral contact lens trial or to follow-up previously issued lenses. They found that the major contact lens indication groups were keratoconus or other primary corneal ectasia, corneal transplant, and ocular surface disease. $^{2}$ The great majority of lenses in use were non-ventilated RGP designs, providing excellent visual results and tolerance. ${ }^{2}$ The design fitted in our cases was also non-ventilated and no anterior segment alterations were detected after the fitting. It should be considered that some studies have previously confirmed that the corneal metabolism is satisfactory with full scleral contact lenses without fenestrations that are made of the currently available materials (high oxygen permeability).$^{7-9}$ Segal et $\mathrm{al}^{10}$ also reported good outcomes in 66 irregular corneas fitted with scleral lenses with a diameter ranging from 15.0 to $24.0 \mathrm{~mm}$. The most common indication for fitting in their sample was keratoconus patients with previous intolerance to other types of corneal lenses. ${ }^{10}$

Besides Pullum et $\mathrm{al}^{2}$ and Segal et $\mathrm{al}^{10}{ }^{10}$ other authors have also reported good results with other designs of full scleral contact lenses, concluding in most of cases that these lenses are a good alternative for patients with corneal abnormalities who failed or became intolerant to other types of contact lenses. ${ }^{3,4}$ This also happened in our case, an advanced keratoconus eye with some previous unsatisfactory contact lens fittings. The main reason for the high level of comfortability achieved with a full scleral lens is that it cannot be dislodged by the lids and the lid margin is in contact with the surface of the lens rather than the edge. ${ }^{2}$ Likewise, it should be considered that full scleral lenses do not touch the cornea, only resting on the conjunctiva over the sclera, which is a less sensitive structure to mechanical stimuli than the cornea. ${ }^{11}$ Therefore, full scleral contact lenses may be considered as a first option to fit in highly irregular corneas in order to minimize the number of tries, but also in less irregular corneas as they are a comfortable and safe contact lens option. Future studies reporting the long-term outcomes of full scleral lenses should confirm if this comfortability and safety is maintained over time. Schornack and Patel $^{5}$ reported an acceptable visual acuity and comfort in patients with keratoconus and fitted with a specific modality of scleral contact lens during a follow-up 
up to 32 months. Visser et $\mathrm{al}^{6}$ found that irregular corneas (mostly keratoconus) fitted with scleral lenses had a comfortable wearing during a 5 month follow-up, with most of them (79.2\%) maintaining the same lens parameters. In our case, the follow-up was of 1 year and the patient preserved the visual acuity achieved and had a comfortable wearing.

\section{CONCLUSION}

Fully scleral contact lenses may be a good option in advanced keratoconus, especially if pre-vious fittings with other types of contact lenses have failed. This type of lenses is able to provide a significant increase in visual acuity combined with a significant improvement in visual quality, maintaining high levels of comfortability. The fitting process of these lenses is relatively simple and can be facilitated significantly with the use of optical coherence tomography to evaluate the central corneal clearance. More studies are necessary in the future to evaluate the results of the scleral lens evaluated in the long-term, including the analysis of other variables, such as corneal endothelial cell density, patient's satisfaction evaluated with a validated questionnaire or the level of contrast sensitivity achieved.

\section{REFERENCES}

1. Van der Worp E, Bornman D, Ferreira DL, Faria-Ribeiro M, Garcia-Porta N, González-Meijome JM. Modern scleral contact lenses: a review. Cont Lens Anterior Eye 2014;37(4):240-250.

2. Pullum KW, Whiting MA, Buckley RJ. Scleral contact lenses: the expanding role. Cornea 2005;24(3):269-277.

3. Pecego M, Barnett M, Mannis MJ, Durbin-Johnson B. Jupiter scleral lenses: the UC Davis Eye Center experience. Eye Contact Lens 2012;38(3):179-182.

4. Rathi VM, Mandathara PS, Dumpati S, Vaddavalli PK, Sangwan VS. Boston ocular surface prosthesis: an Indian experience. Ind J Ophthalmol 2011;59(4):279-281.

5. Schornack MM, Patel SV. Scleral lenses in the management of keratoconus. Eye Contact Lens 2010;36(1):39-44.

6. Visser ES, Visser R, van Lier HJ, Otten HM. Modern scleral lenses part I: clinical features. Eye Contact Lens 2007;33(1):13-20.

7. Bleshoy H, Pullum KW. Corneal response to gas permeable impression scleral lenses. J Br Contact Lens Assoc 1988;11:31-34.

8. Pullum KW, Hobley AJ, Davison C. 100 + DK: Does thickness make much difference? J Br Contact Lens Assoc 1991;6:158-161.

9. Pullum KW, Stapleton FJ. Scleral lens induced corneal swelling: what is the effect of varying Dk and lens thickness? CLAO J 1997;23(4):259-263.

10. Segal O, Barkana Y, Hourovitz D, Behrman S, Kamun Y, Avni I, Zadok D. Scleral contact lenses may help where other modalities fail. Cornea 2003;22(4):308-310.

11. Navascues-Cornago M, Maldonado-Codina C, Morgan PB. Mechanical sensitivity of the human conjunctiva. Cornea 2014;33(8):855-859. 